\title{
Actividad antibacteriana y antioxidante del hongo Phanerochaete spp.
}

\author{
Yessy C. Mena ${ }^{1}$, Luz S. Ramírez ${ }^{1^{\star}}$ y Ángela M. Arango ${ }^{2}$ \\ (1) Facultad de Tecnología, Grupo polifenoles, Escuela de Química, Universidad Tecnológica de Pereira, Pereira- \\ Risaralda-Colombia (correo-e: yessy19utp.edu.co, luramire@utp.edu.co). \\ (2) Facultad de Ciencias de la Salud, Programa de Microbiología, Universidad Libre Seccional Pereira, Sede Belmonte, \\ Pereira-Risaralda-Colombia (correo-e: angela040896@hotmail.com).
}

* Autor a quien debe ser dirigida la correspondencia.

Recibido Jun. 19, 2020; Aceptado Ago. 19, 2020; Versión final Oct. 10, 2020, Publicado Feb. 2021

\begin{abstract}
Resumen
El objetivo de esta investigación fue determinar la capacidad antioxidante y antibacteriana in-vitro de extractos del hongo Phanerochaete spp. Se hicieron tres variaciones en los fermentos: 1) extracto de acetato de etilo, 2) extracto liofilizado y 3) biomasa seca. La actividad antibacteriana se evaluó por la técnica Kirby-Bauer. La actividad antioxidante se examinó por los métodos de Capacidad de Absorción de Radicales Oxigeno (ORAC, en inglés) y DPPH (2,2-difenil-1-picrilhidracilo). La muestra en acetato de etilo presentó actividad antibacteriana frente a cepas de Staphylococcus aureus, con inhibición de $54.76 \%$. La muestra liofilizada presentó la mayor actividad antioxidante con valores de $36.59 \pm 3.53$ y $30.61 \pm 0.20 \mu \mathrm{M}$ Trolox/g. El extracto en acetato de etilo obtuvo más del $50 \%$ de compuestos flavonoides (531 $\pm 4.24 \mu \mathrm{M} \mathrm{EC/g}$ de extracto) en relación con la muestra liofilizada y la biomasa seca. Se concluye que el mejor procedimiento para la obtención de metabolitos secundarios de Phanerochaete spp. fue la extracción con acetato de etilo.
\end{abstract}

Palabras clave: antibacteriana; DPPH; Phanerochaete spp.; ORAC

\section{Antibacterial and antioxidant activity of the fungus Phanerochaete spp.}

\begin{abstract}
The main objective of this research study was to determine in-vitro antioxidant and antibacterial capacity of extracts from the fungus Phanerochaete spp. Three different ferments were examined: 1 ) ethyl acetate extract, 2) a lyophilized extract, and 3) dry biomass. Antibacterial activity was evaluated by the Kirby-Bauer technique. Antioxidant activity was measured by using the ORAC (Oxygen Radical Absorption Capacity) and DPPH (2,2diphenyl-1-picrylhydracil) methods. The ethyl acetate extract showed antibacterial activity against Staphylococcus aureus strains, with an inhibition of $54.76 \%$. The lyophilized extract had the most antioxidant activity with values of $36.59 \pm 3.53$ and $30.61 \pm 0.20 \mu \mathrm{M}$ Trolox/g. The ethyl acetate extract had more than $50 \%$ of flavonoid compounds ( $531 \pm 4.24 \mu \mathrm{M} \mathrm{EC/g}$ of extract) when compared to that of lyophilized and dry biomass samples. It is concluded that the best procedure for obtaining secondary metabolites from Phanerochaete spp. was extraction with ethyl acetate.
\end{abstract}

Keywords: antibacterial; DPPH; Phanerochaete spp.; ORAC 


\section{INTRODUCCIÓN}

Los hongos son fuente de una amplia variedad de metabolitos, muchos de los cuales aún no han sido identificados ni caracterizados, también han sido uno de los mayores patógenos que afectan las plantas, y el afán por eliminar estas enfermedades en los cultivos ha llevado a un alto uso de compuestos químicos para su erradicación. Se ha observado un aumento en la resistencia de los patógenos fúngicos, lo que ha llevado a la necesidad de investigar el potencial antimicrobiano de dichos endófitos (Khruengsai et al., 2019). Por otro lado, existe una relación mutualista entre la planta hospedera y los hongos endófitos ya que estos pueden contribuir a la protección de la planta, mediante la producción de diferentes metabolitos secundarios. (Pansanit and Pripdeevech, 2018). En los últimos años, esa relación mutualista ha sido atribuida a factores como la coevolución de rutas biosintéticas, por ello es muy interesante la búsqueda de nuevos compuestos con potenciales antibacterianos a partir de fuentes endófitas. (Kyekyeku et al., 2017).

Diversos grupos de hongos son fuente de metabolitos como alcaloides, benzoquinonas, flavonoides, ácidos orgánicos, fenoles, esteroides entre otros. Algunos de estos metabolitos podrían ejercer diferentes actividades biológicas, entre ellas la antioxidante (Chandra, Sharma and Arora, 2020). Se han reportado metabolitos secundarios con acción antibiótica, antimicrobiana, anticancerígena, antiproliferativa, anti-inflamatoria, entre otros (Sahu et al., 2020). Es así como el hongo Phanerochaete ha sido estudiado en el ámbito de la producción de enzimas peroxidasas, lignina-peroxidasa, y manganeso peroxidasa, importantes en la degradación de la lignina, sin embargo, existe muy poca información respecto al potencial como agente antibacteriano y antioxidante de este hongo.

El uso indiscriminado de antibióticos a nivel mundial ha ido generando resistencia de los microorganismos a estos tratamientos, lo que se ha convertido en un tema de interés para la salud pública. (Montero-Recalde et al., 2018). La infección microbiana es una de las principales causas de mortalidad en todo el mundo y los productos naturales han demostrado un papel fundamental en la lucha contra esas enfermedades infecciosas, por ello es importante la búsqueda de material vegetal que tenga algún potencial antimicrobiano. A pesar de esto menos del $5 \%$ del total de la diversidad de las plantas han sido analizadas en cuanto a su contenido endófito y menos aún en cuanto a los productos naturales y sus bioactividades (Kapoor, Jamwal and Gandhi, 2019)

La diversidad biológica encontrada en Colombia en las últimas décadas, ha sido objeto de estudio puesto que se han hallado microorganismos productores de sustancias con actividad antimicrobiana, antiviral, anticancerígena, entre otras (Elvira et al., 2013), ya que por su ubicación geográfica, tipos de vegetación y clima, presenta las condiciones suficientes para que los hongos puedan producir una diversidad importante de sustancias químicas, generando con ello un interés medicinal como compuestos antibacterianos y antioxidantes principalmente, puesto que desde hace varios años algunas cepas bacterianas han desarrollado resistencia a los antibióticos disponibles, cifras de la Organización Mundial de la Salud (OMS) declaran que alrededor del $26 \%$ de la mortalidad global se debe a enfermedades infecciosas de los cuales, alrededor del $87 \%$ lo representan las infecciones microbianas. Paralelamente, la OMS reportó que, las enfermedades crónicas no transmisibles conforman la principal causa de muerte en todo el mundo, posiblemente atribuido a un desequilibrio respecto a los agentes radicalarios, desencadenando un fenómeno llamado estrés oxidativo que origina distintas reacciones desfavorables en las células (Cortes, 2017).

Phanerochaete spp. es conocido como "Hongo xilófago" debido a que descompone la madera (pudrición blanca), especialmente los compuestos lignocelulósicos generando $\mathrm{CO}_{2}$ y $\mathrm{H}_{2} \mathrm{O}$; gracias a la segregación de enzimas como celulasas, hemicelulasas, xilanasas, LiP, MnP y lacasa (Vega and Franco, 2013), por consiguiente, desempeña un papel importante en la descomposición de los tejidos de las plantas, así como en la obtención de productos como bioetanol, al degradar la celulosa en moléculas de glucosa (Ferreira, 2009). El estudio de los metabolitos secundarios del hongo Phanerochaete spp. es muy escaso en la actualidad, por tanto, el presente trabajo es un estudio preliminar de compuestos bioactivos obtenidos por fermentación de este hongo tendiente a explorar su potencial como antibacteriano y antioxidante mediante técnicas como difusión en pozo, ORAC y DPPH

\section{METODOLOGÍA}

El procedimiento desarrollado para la obtención de la muestra liofilizada, biomasa seca y extracto en acetato de etilo para evaluar las actividades biológicas mencionadas se describe a continuación.

\section{Recolección, aislamiento y purificación del hongo Phanerochaete spp.}

El cuerpo fructífero del hongo Phanerochaete spp. fue recolectado en el Jardín Botánico de la Universidad Tecnológica de Pereira y se almacenó en el laboratorio de Microbiología y actividad biológica de la escuela 
de Química. Éste se sometió a un tratamiento de desinfección empleando la metodología descrita por Suárez, C. (Suárez, 2010) y se sembró en el medio de cultivo Sabouraud por triplicado a temperatura ambiente en oscuridad. Se realizó un seguimiento macro y microscópico para observar variaciones morfológicas empleando azul de lactofenol

\section{Fermentaciones fúngicas de Phanerochaete spp.}

Tras la purificación del hongo, se llevaron a cabo tres metodologías diferentes de fermentación para la obtención de los metabolitos secundarios, éstas consistieron en: i) Fermento 1: El cultivo de siembra se inoculó con $1 \mathrm{~mL}$ de esporas, previamente sembradas en $10 \mathrm{~mL}$ de medio PDA al 5\%. El pH se ajustó a 5.5 y el proceso tuvo una duración de 15 días. El medio liquido estaba compuesto por: Peptona bacteriológica $4 \%$, aceite de oliva $1 \%$, sacarosa $0.5 \%, \mathrm{MgSO}_{4} 7 \mathrm{H}_{2} \mathrm{O} 0.1 \%, \mathrm{KH}_{2} \mathrm{PO}_{4} 0.1 \%$ y $\left(\mathrm{NH}_{4}\right)_{2} \mathrm{SO}_{4} 0.1 \%$ (Sharma, 2017); ii) Fermento 2: $60 \mathrm{~mL}$ de PDA al $5 \%$ (caldo fermentativo) fue inoculado con $20 \mathrm{~mL}$ de suspensión de esporas a una concentración de $9.12 \times 10^{6}$ conidios $/ \mathrm{mL}$, y iii) Fermento 3: El fermento PDB utilizado contenía papa, sacarosa y agua destilada adaptando el método de Indarmawan (2016) para su preparación. El caldo de cultivo se inoculó con varias fracciones de micelio fresco, sembrado con 8 días de anterioridad en PDA. La fermentación tuvo una duración de 17 días. Los cultivos durante el proceso de fermentación se mantuvieron en un shaker en oscuridad a $28^{\circ} \mathrm{C}$ y $150 \mathrm{rpm}$. Las condiciones de la fermentación se muestran en la tabla 1.

Tabla 1. Condiciones para la fermentación del hongo Phanerochaete

\begin{tabular}{|c|l|l|l|}
\hline Muestra & \multicolumn{1}{|c|}{ Condiciones del inóculo } & \multicolumn{1}{|c|}{ Tiempo de fermentación } & \multicolumn{1}{c|}{ Caldo } \\
\hline 1 & $1 \times 10^{6}$ Esporas $/ \mathrm{mL}$ & 15 días & PDA $5 \%$ \\
\hline 2 & $20 \mathrm{~mL}$ de esporas a $12 \times 10^{6}$ conidios $/ \mathrm{mL}$ & 15 días & PDA $5 \%$ \\
\hline 3 & Fracción del micelio fresco & 17 días & PDB \\
\hline
\end{tabular}

Al finalizar el proceso de fermentación, el medio de cultivo de cada muestra fue filtrado y la biomasa retenida fue secada al horno durante 24 horas a $60^{\circ} \mathrm{C}$ (Chaparro, 2010). El filtrado fue distribuido en dos alícuotas; una para ser liofilizada y la otra para obtener un extracto en acetato de etilo 1:1 que posteriormente se llevó a sequedad por rota-evaporación.

Como parte del seguimiento microscópico y cromatográfico cada cinco días se tomó una alícuota del caldo de cultivo para realizar observaciones al microscopio, placas cromatográficas en diferentes fases móviles: nhexano-acetato de etilo (7:3) y cloroformo-metanol (9:1), se observó bajo la luz ultravioleta a onda corta (254 $\mathrm{nm})$ y larga $(365 \mathrm{~nm})$ además de los siguientes reveladores mostrados en la tabla 2

Tabla 2 Pruebas fitoquímicas preliminares en placa CCF para el extracto en acetato de etilo.

\begin{tabular}{|l|l|l|}
\hline Revelador & Núcleo Fitoquímico & Tonalidad \\
\hline $\mathrm{AlCl}_{3}$ & Flavonoides. & Manchas amarillas fluorescentes \\
\hline Borntrager (KOH/EtOH) & Antraquinonas, Antronas y Cumarinas & Manchas azul fluorescente \\
\hline Liebermann-Burchard & Esteroides y terpenos & Manchas rojo-rosado-purpura para triterpenos \\
\hline Óleum & Lactonas sesquiterpénicas & Manchas rojas o rosadas \\
\hline Vainillina 5\% & Saponinas & Mancha azul, violeta o amarilla \\
\hline $\mathrm{FeCl}_{3}$ & Compuestos fenólicos y/o taninos & $\begin{array}{l}\text { Mancha azul para taninos gálicos y verde para } \\
\text { taninos catéquicos }\end{array}$ \\
\hline Dragendorff & Alcaloides & Manchas rojas-naranjas \\
\hline
\end{tabular}

\section{Evaluación de actividad antibacteriana del hongo Phanerochaete spp.}

La actividad antibacteriana de los extractos obtenidos (Extracto liofilizado, solución obtenida a partir de la biomasa y extracto en acetato de etilo), fue evaluada usando la técnica Kirby-Bauer modificada, mediante la utilización de pozos en agar. (Montero-Recalde et al., 2018) y concentraciones de $2 \mathrm{ug} / \mathrm{mL}, 0.5 \mathrm{ug} / \mathrm{mL}$ y 0.1 $\mathrm{ug} / \mathrm{mL}$ de cada uno de los extractos. Las cepas evaluadas fueron Staphylococcus aures (ATCC 25923), Escherichia coli (ATCC25922) y Pseudomonas aeruginosa (ATCC 27853) sembradas 24 horas antes del ensayo en agar nutritivo (Difco TM 21300). Para obtener la concentración del inóculo bacteriano se utilizó el DensiCHEK Plus; se diluyó en agua estéril la colonia a evaluar hasta obtener una concentración de 0,5

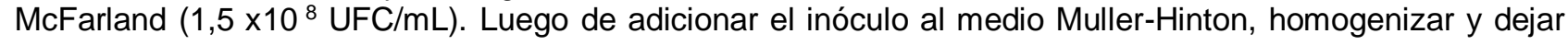


gelificar en las cajas Petri, se realizaron pozos con una pipeta pasteur donde se depositó $20 \mathrm{uL}$ de cada extracto a evaluar y el control positivo correspondiente para cada cepa bacteriana, estos fueron antibióticos comerciales; para Escherichia coli Amoxicilina a $200 \mathrm{ug} / \mathrm{mL}$, para Staphylococcus aureus Ampicilina a $200 \mathrm{ug} / \mathrm{mL}$ y para Pseudomonas aeruginosa Ciprofloxacina a $25 \mathrm{ug} / \mathrm{mL}$. Los ensayos se realizaron por triplicado y se usó la siguiente ecuación 1 (Ramirez , 2015), para determinar el \% de inhibición.

$$
\% \text { inhibición }=\frac{\text { halo de inhibición de la muestra-halo de inhibición del blanco de muestra }}{\text { halo de inhibición de control positivo-halo de inhibición de la muestra }} \times 100
$$

\section{Contenido de flavonoides totales}

La determinación del ensayo se llevó a cabo en una microplaca de 96 pozos utilizando $15 \mu \mathrm{L}$ de $\mathrm{NaNO}_{2}$ al $5 \%$ (excepto el blanco), se mezcló con $100 \mu \mathrm{L}$ de las muestras de biomasa seca, liofilizado y extracto en acetato de etilo a concentraciones entre 62.5 a $2000 \mathrm{mg} / \mathrm{mL}$, seguido de $15 \mu \mathrm{L}$ de $\mathrm{AlCl}_{3}$ al $10 \%, 70 \mu \mathrm{L}$ de $\mathrm{NaOH} 1 \mathrm{M}$. La absorbancia se leyó en un lector de microplacas (Thermo multiscan Go) a los 5 minutos a $500 \mathrm{~nm}$. Se empleó como estándar para la curva de calibración catequina en un rango de concentraciones de 1 a 70 $\mathrm{mg} / \mathrm{mL}$ y se expresó en $\mu \mathrm{M}$ de equivalente de catequina/g de extracto ( $\mu \mathrm{M} \mathrm{EC/g} \mathrm{extracto)} \mathrm{(Ramirez,} \mathrm{2015).}$

\section{Contenido de fenoles totales}

El contenido de fenoles totales en las muestras de biomasa seca, liofilizado y extracto en acetato de etilo se evaluaron según el método de Folin-Ciocalteu modificado, descrito por Magalhães L. (Magalhães, 2010). En la metodología se usó $50 \mu \mathrm{L}$ de cada muestra, $75 \mu \mathrm{L}$ de reactivo de Folin y $75 \mu \mathrm{L}$ de $\mathrm{Na}_{2} \mathrm{CO}_{3}$ al $7.5 \%$, permanecieron 60 minutos en la oscuridad y se leyó la absorbancia en un lector de microplacas (Thermo Multisank GO) a $760 \mathrm{~nm}$. Los compuestos fenólicos se cuantificaron mediante una curva de referencia de ácido gálico a concentraciones entre 1 y $70 \mathrm{mg} / \mathrm{mL}$ y se expresaron como $\mu \mathrm{M}$ de equivalente de ácido gálico/g de extracto ( $\mu \mathrm{M}$ de $E A G / g$ de extracto).

\section{Evaluación de la actividad antioxidante}

La evaluación de la actividad antioxidante del hongo Phanerochaete spp. se determinó mediante las siguientes técnicas:

\section{Ensayo de DPPH}

El estudio de la actividad captadora del radical 2,2-difenil-1-picrilhidracilo ( $\mathrm{DPPH} \bullet$ ) se efectuó de acuerdo con el método propuesto por Brand-Williams et al. con ligeras modificaciones. Para el ensayo se preparó una solución de 2,2-difenil-1-picrilhidracilo en metanol a $20 \mathrm{mg} / \mathrm{mL}$. A un volumen de $25 \mathrm{uL}$ de los extractos obtenidos del hongo Phanerochaete spp (solución de biomasa, extracto en acetato de etilo, y extracto liofilizado) se adicionó 100 uL de solución DPPH. La reacción se desarrolló a temperatura ambiente durante 30 minutos en oscuridad. La lectura de las absorbancias se realizó a $517 \mathrm{~nm}$ con un lector de microplacas. Con las absorbancias manifestadas se calculó el porcentaje de actividad inhibitoria a partir de la ecuación 1 (Ramirez 2015). El control positivo fue Hidroquinona a $1000 \mathrm{mg} / \mathrm{mL}$ y el control negativo fue metanol.

$$
\% \mathrm{~A} . \mathrm{A}=\frac{\mathrm{A}_{\mathrm{c}(-)}-\left(\mathrm{A}_{\text {extracto }}-\mathrm{A}_{\text {blanco extracto }}\right)}{\mathrm{A}_{\mathrm{c}(-)}} * 100
$$

Donde Ac(-) corresponde a la absorbancia del control negativo, Aextracto corresponde a la absorbancia del extracto evaluado, A blanco extracto corresponde a la absorbancia del metanol.

\section{Ensayo de ORAC}

La actividad antioxidante por el método de Capacidad de Absorción de radicales Oxígeno (ORAC) estuvo basada en el método descrito por K. M. Gillespie (Gillespie, Chae and Ainsworth, 2007). Este ensayo se realizó en microplacas de 96 pozos, en cada uno de los pozos se llevó a cabo una reacción que contenía $20 \mu \mathrm{L}$ de cada extracto a evaluar y $120 \mu \mathrm{L}$ de Fluoresceina a una concentración de $200 \mu \mathrm{M}$. La mezcla se dejó incubando durante 15 minutos en oscuridad a $37^{\circ} \mathrm{C}$, pasado este tiempo se adicionó a cada pocillo una solución de AAPH (diclorhidrato de 2,2'-azobis (2-amidinopropano)) a 12 mM. Cada 120 segundos se registró la intensidad de la fluorescencia, usando una longitud de onda de excitación de $485 \mathrm{~nm}$ y $520 \mathrm{~nm}$ de emisión (Boxin Ou, 2001). La actividad antioxidante se expresó como $\mu \mathrm{M}$ equivalente Trolox/g extracto ( $\mu \mathrm{M} \mathrm{ET} / \mathrm{g}$ extracto). 


\section{RESULTADOS Y DISCUSIÓN}

Los resultados obtenidos para el hongo Phanerochaete spp. respecto a las actividades biológicas se indican a continuación:

\section{Aislamiento y purificación del hongo Phanerochaete spp.}

A partir de la purificación del hongo se realizó una caracterización macroscópica donde se observó un micelio con una apariencia algodonosa, aplanada de tonalidad blanca y bordes irregulares (Figura $1^{\mathrm{a}-\mathrm{b}}$ ). Con el tiempo, el micelio se tornó gris y segregó un líquido de color amarillo, alrededor del hongo inicialmente sembrado (Figura $1^{\mathrm{b}}$ ). Es importante anotar que en estudios posteriores se realizó el proceso de identificación molecular, y sus secuencias están en el genbank (MK457296-97-98)

Su crecimiento disminuyó con la replicación constante del hongo, aun así, su morfología macro y microscópica guardaron las características propias del género. Dicha disminución puede ser consecuencia de diversos factores externos como: el clima, la temperatura y la edad del hongo según Orlandelli et al. (Orlandelli et al., 2012)

a.

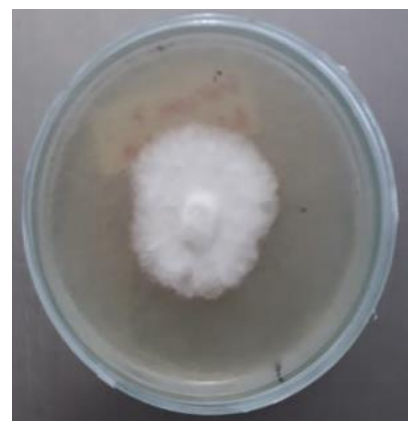

b.

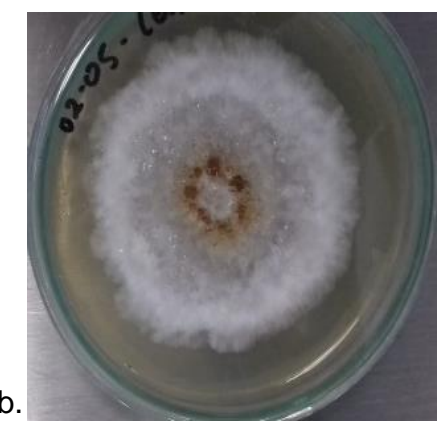

Fig. 1. Hongo Phanerochaete spp. en PDA. a. Crecimiento micelial en 15 días. b. Crecimiento micelial en 2 meses aproximadamente.

De acuerdo con su estructura microscópica, el cuerpo fructífero presenta un sistema hifal dimítico correspondiente a hifas generativas principalmente septadas y esqueléticas de paredes delgadas a ligeramente gruesas, las cuales le brindan soporte al micelio del hongo (Figura $2^{\mathrm{a}}$ ) (Spirin, 2017). De igual forma, se encuentran parcialmente aglomeradas de forma paralela y con frecuencia ramificada (Martinez 2005). Así mismo, se observaron dos basidiosporas que se forman tras un proceso de reproducción sexuada en el interior de un basidio (figura $2^{b}$ )

a.

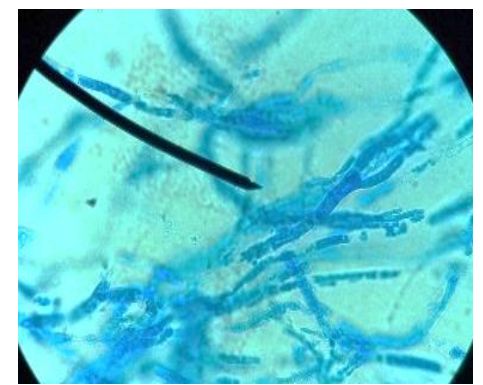

b.

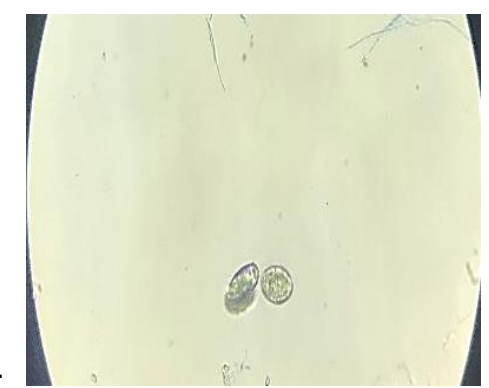

Fig. 2: Morfología microscópica del hongo Phanerochaete spp. a. Hifas. b. Basidiosporas.

Proceso fermentativo e identificación de metabolitos secundarios del hongo Phanerochaete spp.

La tabla 3 señala la presencia de algunos metabolitos secundarios caracterizados desde el punto de vista cualitativo, mediante cromatografía de capa delgada en silica gel. La identificación de núcleos fitoquímicos como terpenos y cumarinas se evidencian por la presencia de coloraciones intensas azules, rojas y rosadas las cuales fueron observadas en la placa cromatográfica al momento de revelarse con el reactivo de Liebermann-Burchard y Borntrager $(\mathrm{KOH} / \mathrm{EtO})$ respectivamente. Estos resultados son similares a los compuestos activos identificados en un extracto obtenido del hongo Phanerochaete spp por Khruengsai et al, quienes reportan la presencia de compuestos como perileno, benzoina, collumeralina, totarena y stemona, a los que se atribuye capacidad antimicrobiana (Khruengsai et al., 2019); estos compuestos son clasificados dentro del grupo de los monoterpenos, sesquiterpenos y sus derivados, además de la evidencia de terpenos y antraquinonas (Liu et al., 2019) (Qi et al., 2020). 
En los resultados cromatográficos también se observó la presencia de metabolitos como flavonoides y fenoles, los cuales se asocian con la formación de complejos con las proteínas solubles y la pared bacteriana generando inactivación de dicha pared (Cuadros, 2010), estos metabolitos junto con las cumarinas se caracterizan por su buena capacidad antioxidante frente a los radicales libres del oxígeno puesto que previenen el daño oxidativo causado por el superóxido $\left(\mathrm{O}^{-2}\right)$ y el radical hidroxilo (HO.) (Avila, 2009); Otros estudios realizados en extractos metanolicos de hongos endófitos como Phyllosticta sp, demostraron tener compuestos fenólicos y flavonoides, los cuales mostraron tener actividad contra radicales ABTS y DPPH (Chandra, Sharma and Arora, 2020) Así mismo, las lactonas sesquiterpénicas conforman dicho grupo de compuestos secundarios conocido por su amplia aplicación como agente antitumoral, antiinflamatorio y por su propiedad de inducir apoptosis (Chaparro, 2010). Por otro lado, se han aislado dos compuestos sesquiterpenos del hongo xylaria $s p$ lo cual se puede relacionar con los compuestos identificados cualitativamente mediante cromatografía de capa delgada (Kapoor, Jamwal and Gandhi, 2019)

Tabla 3. Caracterización preliminar de los metabólitos secundarios del extracto en acetato en etilo. Fase móvil: CloroformoMetanol (9:1). (-): Ausencia del núcleo, (+): Presencia del núcleo y (++): Presencia abundante.

\begin{tabular}{|c|c|c|c|c|}
\hline Prueba & Núcleo Fitoquímico & Fermento 1 & Fermento 2 & Fermento 3 \\
\hline $\mathrm{AlCl}_{3}$ & Flavoniodes & + & - & ++ \\
\hline Borntrager (KOH/EtOH) & Antraquinonas, Antronas y Cumarinas & + & ++ & + \\
\hline Liebermann-Burchard & Esteroides y terpenos & - & ++ & ++ \\
\hline Óleum & Lactonas sesquiterpénicas & + & ++ & ++ \\
\hline Vainillina 5\% & Saponinas & + & + & + \\
\hline $\mathrm{FeCl}_{3}$ & Compuestos fenólicos y/o taninos & + & - & + \\
\hline Dragendorff & Alcaloides & + & + & + \\
\hline
\end{tabular}

\section{Actividad antibacteriana del hongo Phanerochaete spp.}

Como se observa en la tabla 4, los resultados de la actividad antibacteriana del extracto en acetato de etilo, fueron positivos frente a la inhibición del crecimiento de la bacteria Gram Positiva S.aureus, y negativos frente a las bacterias E. coli y P.aeruginosa. El porcentaje de inhibición para S.aureus fue del $54.76 \%$ cuando se expuso el extracto en acetato de etilo a una concentración de $2 \mu \mathrm{g} / \mathrm{mL}$. Estos resultados son acordes con reportes realizados por otros autores donde se evalúa la actividad antibacteriana de varios extractos en acetato de etilo, de hongos endófitos, los extractos mostraron alguna actividad frente a patógenos humanos, pero especialmente contra bacterias Gram positivas. Este comportamiento posiblemente se encuentra asociado a la estructura de la pared celular de la bacteria Gram negativa, la cual está compuesta por lipopolisacáridos hidrofílicos que evitan la penetración de aceites hidrofóbicos, y como consecuencia los extractos en acetato de etilo de hongos endófitos tienen mayor actividad contra bacterias Gram positivas.

Finalmente la actividad antibacteriana de los extractos fúngicos podría ser el resultado de la acción sinérgica de sus compuestos (Pansanit and Pripdeevech, 2018). Esto podría explicarse ya que en otro estudio realizado por Kyekyeku et al, ninguno de los compuestos aislados individualmente, mostraron alguna actividad contra cepas patógenas de S.aureus y B. subtilis (Kyekyeku et al., 2017). Las evaluaciones de actividad antibacteriana para las muestras liofilizadas y materia seca no arrojaron resultados positivos frente a ninguna de las bacterias evaluadas. Por otro lado, la enzima celobiosa deshidrogenasa aislada del hongo Phanerochaete. chrysosporium, evidenció inhibición contra el crecimiento de la cepa $S$. aureus cuando se usa Lactosa como sustrato, y una muy baja inhibición para E. coli y P. aeruginosa. (Sulej et al., 2019), lo que es acorde con los resultados arrojados en este estudio.

Tabla 4. Comparación de los halos de inhibición del extracto en acetato de etilo frente a las cepas bacterianas analizadas.

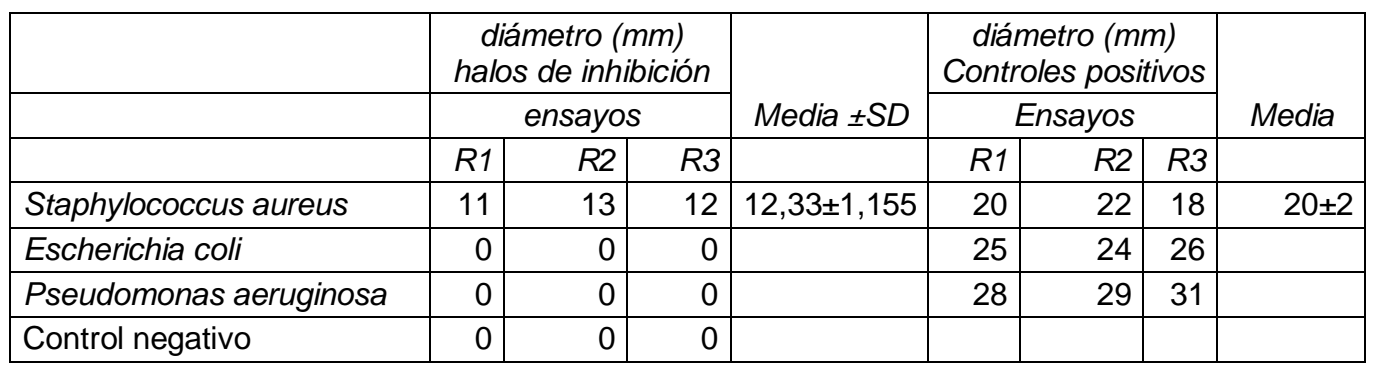




\section{Contenido de flavonoides totales}

Los resultados obtenidos en la cuantificación de contenido total de flavonoides se observan en la figura 3 , oscilando entre $189 \pm 19.33$ y $531 \pm 4.24 \mu \mathrm{M} \mathrm{EC/g}$ de extracto calculados con curva de calibración de catequina (3-241 $\mu \mathrm{M} / \mathrm{L})$. El extracto en acetato de etilo obtuvo más del $50 \%$ de compuestos flavonoides (531 $\pm 4.24 \mu \mathrm{M} \mathrm{EC/g}$ de extracto) en relación con la muestra liofilizada y la biomasa, una de las razones por las cuales se puede presentar este comportamiento se fundamenta en que los compuestos bioactivos se sintetizan dentro de las células fúngicas y son obtenidos por procesos de extracción con solventes (Chandra et al., 2019). Dichos resultados están un poco por encima de los reportados por Ávila I. M. sobre especies basidiomicetos, en el cual sus resultados se encontraron entre 0.02 y $0.01 \mathrm{mg} \mathrm{EC} / \mathrm{g}$ de extracto(Barros et al., 2008).lo que puede atribuirse a que es otra especie de hongo. De acuerdo con investigaciones similares realizadas por Barros, L. para hongos basidiomicetos, las concentraciones halladas coinciden con las encontradas en este trabajo, en términos generales están por debajo del contenido de fenoles totales, lo cual se explica por el hecho que los flavonoides corresponden a una subclase de éstos, en algunos casos, su valor no es suficiente para poseer un aporte antioxidante importante de forma individual, o bien, una actividad pro-oxidativa se contrapone al potencial antioxidante (Hossain et al., 2011). Se ha comprobado que los flavonoides presentan un rango amplio de acciones farmacológicas como actividades antitrombóticas, antimutagénicas y son los agentes antioxidantes predominantes entre los compuestos fenólicos(Barros et al., 2008).

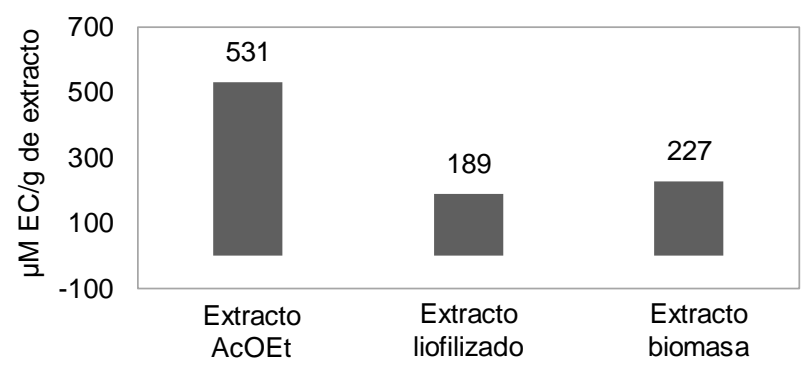

Fig. 3. Contenido de flavonoides totales.

\section{Contenido de fenoles totales.}

En la figura 4 se describen las concentraciones obtenidas, los cuales varían entre 0 y $1679.37 \pm 2.79 \mu \mathrm{M}$ $\mathrm{EAG} / \mathrm{g}$ de extracto donde la muestra en acetato de etilo obtuvo el mayor contenido de fenoles totales, algunos compuestos fenólicos como ácido gálico, ácido ferúlico y ácido ellagico son producidos por diferentes fuentes microbianas bajo fermentación en estado sólido y sumergido y la extracción de estos compuestos se hacen mediante el uso de diferentes solventes orgánicos. (Bhanja Dey et al., 2016). Las especies que reflejaron mayor cantidad de fenoles totales, es decir, el extracto en acetato de etilo y liofilizado reportaron la mejor actividad en DPPH y ORAC, demostrando que los compuestos con diferentes grupos fenólicos en cada molécula, son los que mayor actividad antioxidante poseen, por tanto, presentan una correlación directa entre ellos, lo cual se permite asociar con estudios previos reportados por Barros, L.(Barros et al., 2007). Otro estudio donde se evalúa la capacidad antioxidante del hongo Phanerochaete chrysosporium, indicó que el contenido de fenoles está directamente relacionado con la actividad antioxidante, los compuestos fenólicos de los hongos evaluados estuvieron dentro de un rango de 8 a $16,7 \mathrm{mg} / \mathrm{mL}$ (Chandra et al., 2019). Los hongos de la pudrición blanca tienen una gran cantidad de metabolitos secundarios que hacen parte de todo un espectro de compuestos polifenólicos en forma de fenoles y derivados flavonoides, además de enzimas antioxidantes de bajo peso molecular y polisacáridos. La bioactividad de los compuestos fenólicos puede estar relacionada con su capacidad para quelar los metales, inhibir la lipoxigenasa y eliminar los radicales libres (Kitzberger et al., 2007).En cuanto al extracto de biomasa no presento fenoles totales, lo cual se puede explicar por la presencia de flavonoides no heterocíclicos o metoxilados.

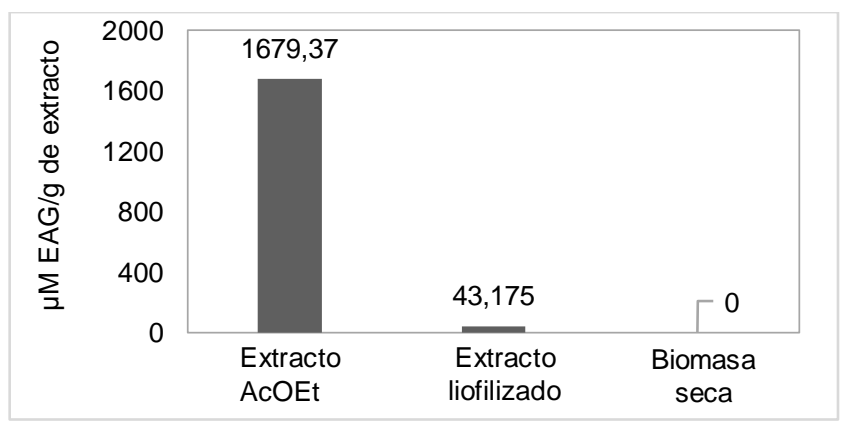

Fig. 4. Contenido de fenoles totales. 
Evaluación de la actividad antioxidante del hongo Phanerochaete spp.

La evaluación de los ensayos DPPH y ORAC arrojaron los siguientes valores a una concentración de $2000 \mathrm{mg} / \mathrm{mL}$ :

\section{Ensayo de DPPH}

Los resultados de este ensayo se muestran en la tabla 5, expresando que el contenido más alto de actividad antioxidante corresponde a la fracción liofilizada con un valor de $30.61 \pm 0.20 \mu \mathrm{M}$ de Trolox/g de extracto mientras que la biomasa seca presentó la más baja capacidad para atrapar radicales DPPH $(13.92 \pm 0.01 \mu \mathrm{M}$ de Trolox/g de extracto). Los valores obtenidos concuerdan con investigaciones reportadas ya que la liofilización tiene la capacidad de conservar al máximo todos los compuestos metabólicos presentes en un fermento (Amzad Hossain and Shah, 2015) De igual manera otros autores como Chandra et al., 2019, encontraron en los extractos filtrados de varios hongos de la pudrición blanca, entre ellos, Phanerochaete chrysosporium efectos significativos contra radicales DPPH con un \% de actividad antioxidante del $72,5 \% \pm 0,4$ (Chandra et al., 2019). Se sugiere que para próximos estudios se evalúen otras variables de fermentación en estado sumergido con el fin de garantizar las condiciones metabólicas que favorezcan la mayor producción de compuestos fenólicos y flavonoides, lo que directamente se verá reflejado en la actividad antioxidante del extracto obtenido por fermentación.

A partir de estos resultados, se puede deducir que la actividad antioxidante de los diferentes extractos no solo se correlaciona con compuestos fenólicos, sino también con otros metabolitos secundarios tales como alcaloides, saponinas, cumarinas que debido a su estructura contribuyen a su eficacia antioxidante (Chao, 2014), por lo tanto los extractos liofilizado y en acetato de etilo se consideran como promisorios en cuanto a su capacidad de captación de radicales.

Tabla 5. Ensayo DPPH a una concentración de $2000 \mathrm{mg} / \mathrm{mL}$.

\begin{tabular}{|l|c|c|}
\hline Muestras & $\%$ A.A & $\mu M$ Trolox $/ g$ \\
\hline Extracto en acetato de etilo & $42.77 \pm 1.81$ & $25.75 \pm 0.02$ \\
\hline Liofilizada & $53.27 \pm 0.21$ & $30.61 \pm 0.20$ \\
\hline Biomasa seca & $17.79 \pm 1.34$ & $13.92 \pm 0.01$ \\
\hline Control positivo (Hidroquinona $1000 \mathrm{mg} / \mathrm{mL}$ ) & $60.89 \pm 2.37$ & $68.75 \pm 0.35$ \\
\hline
\end{tabular}

\section{Ensayo de ORAC}

Los resultados obtenidos se expresan en la tabla 6 , señalando que la muestra liofilizada presentó la mayor actividad antioxidante con una concentración de $36.59 \pm 3.53 \mu \mathrm{M}$ de Trolox/g de extracto. Con base en estudios reportados, este ensayo depende directamente del contenido de flavonoides y su actividad se debe principalmente a sus propiedades redox, es decir, depende de su estructura química y su capacidad de donar o aceptar electrones, deslocalizando así un electrón no apareado dentro de una estructura aromática (Belloso, 2015). Las mediciones de ORAC se han convertido en una de las actividades biológicas más relevantes puesto que los radicales peroxilo son los más comunes encontrados en el cuerpo humano (Zaragoza, 2011).

Tabla 6. Ensayo ORAC a una concentración de $2000 \mathrm{mg} / \mathrm{mL}$.

\begin{tabular}{|l|c|}
\hline Muestras & $\mu M$ Trolox $/ \mathrm{g}$ \\
\hline Extracto en acetato de etilo & $19.01 \pm 4.30$ \\
\hline Liofilizada & $36.59 \pm 3.53$ \\
\hline Biomasa seca & $25.33 \pm 2.69$ \\
\hline Control positivo (Quercetina $2.5 \mathrm{mg} / \mathrm{mL}$ ) & $22734.64 \pm 1834.49$ \\
\hline
\end{tabular}

\section{CONCLUSIONES}

El método más adecuado para la obtención de metabolitos secundarios después de la fermentación, fue la extracción con acetato de etilo; mostrando que el hongo Phanerochaete spp. es una fuente de compuestos secundarios tales como alcaloides, flavonoides, saponinas, esteroides y terpenos, los cuales han sido reconocidos por su potencial biológico. Se encontró una relación directa entre el contenido de flavonoides y fenoles totales con la actividad antioxidante, puesto que la muestra liofilizada y en acetato de etilo presentaron el mayor porcentaje de actividad al igual que el mayor contenido de flavonoides y fenoles totales 
El extracto en acetato de etilo del hongo Phanerochaete spp exhibe actividad antibacteriana contra Staphylococcus aureus ATCC 25923. Dicho extracto al igual que el extracto liofilizado presentaron actividad antioxidante por los dos métodos evaluados. Es necesario continuar realizando ensayos de caracterización de compuestos flavonoides y fenólicos para confirmar la correlación existente entre las estructuras y la actividad antioxidante, al igual que la correlación existente entre lactonas sesquiterpenicas, terpenos y otros compuestos responsables de la actividad antibacteriana.

Los resultados obtenidos constituyen un primer estudio exploratorio de caracterización biológica del hongo Phanerochaete spp. por tal razón, es necesario complementarlo con otros estudios, tanto desde el punto de vista toxicológico como de sus componentes activos, con el objeto de ser aplicado en futuro a nivel industrial, en la rama farmacológica, principalmente.

\section{REFERENCIAS}

Amzad-Hossain, M. and Shah, M. D. 'A study on the total phenols content and antioxidant activity of essential oil and different solvent extracts of endemic plant Merremia borneensis', doi: 10.1016/j.arabjc.2011.01.007Arabian Journal of Chemistry, 8(1), pp. 66-71. (2015)

Barros, L, Falcao S, Baptista y otros tres autores. 'Total phenols, ascorbic acid, $\beta$-carotene and lycopene in Portuguese wild edible mushrooms and their antioxidant activities', doi: 10.1016/j.foodchem.2006.07.038. Food Chemistry, 103(2), pp. 413-419. (2007)

Belloso, K., González, I., Suaréz, R. Actividad antioxidante de extractos de diez basidiomicetos comestibles en Guatemala', https://digi.usac.edu.gt/ojsrevistas/index.php/cytes/article/view/65 Ciencia, Tecnologí-a y Salud, 2(2), pp. 2936 (2015)

Bhanja-Dey, T.Chakraborty, S., y otros tres autores. Antioxidant phenolics and their microbial production by submerged and solid state fermentation process: A review', doi: 10.1016/j.tifs.2016.04.007.Trends in Food Science and Technology, 53, pp. 60-74. (2016)

Boxin, O., Hamspsch-Woodill, M., Prior, R. L. doi: 10.1051/jp2:1995126. (Development and Validation of an Improved Oxygen Radical Absorbance Capacity Assay Using Fluorescein as the Fluorescent Probe', Food Chemistry, 49(2), pp. 4619-4625. (2001)

Chandra, P., Sharma, R. K. y Arora, D. S. Antioxidant compounds from microbial sources: A review', doi: 10.1016/j.foodres.2019.108849.Food Research International, 129(November 2019), p. 108849. (2020)

Chaparro, A. P 'Aislamiento e identificaciòn de metabolitos producidos por la cepa nativa spg 321 de', pp. 1-116. Available at: https://javeriana.edu.co/biblos/tesis/ciencias/tesis389.pdf. (2010)

Cortes Meza, S., Ortiz, A. y Ramirez, L. S.'Determinación de antioxidante en subproductos de café producido y comercializado en Risaralda (Colombia)., Pereira: Universidad Tecnológica de Pereira, pp. 1-8. Available at: http://repositorio.utp.edu.co/dspace/bitstream/handle/11059/7597/63373C828.pdf?sequence=1. (2017)

Cuadros, A. M.Evaluación antibacteriana de metabolitos secundarios de extractos de cepas híbridas de Pleurotus spp .', Dissertação. (2010)

Elvira, R. B. Lorena., y otros tres autores. Hongos endófitos: fuente potencial de metabolitos secundarios bioactivos con utilid ad en agricultura y medicina', Revista especializada en Ciencias Químicos-biológicas, 16(2), pp. 132-146. (2013)

Gillespie, K. M., Chae, J. M. y Ainsworth, E. Rapid measurement of total antioxidant capacity in plants doi: 10.1038/nprot.2007.100. Nature protocols, 2(4), pp. 867-70. (2007)

Hossain, M., Shah, M., Gnanaraj, C., y Muhammad I. 'In vitro total phenolics, flavonoids contents and antioxidant activity of essential oil, various organic extracts from the leaves of tropical medicinal plant Tetrastigma from Sabah', doi: 10.1016/S1995-7645(11)60180-6. Asian Pacific Journal of Tropical Medicine, 4(9), pp. 717-721. (2011)

Indarmawan, T. Muatopa, A., Budiarto, B., Tarman K. Antibacterial Activity of Extracellular Protease Isolated From an Algicolous Fungus Xylaria psidii KT30 Against Gram-Positive Bacteria' doi: 10.1016/j.hjb.2016.06.005(2016)., HAYATI Journal of Biosciences, 23(2), pp. 73-78. (2016)

Kpoor, N., Jamwal, V. L. y Gandhi, S. G. Endophytes as a Source of High-Value, Bioactive Metabolites. doi: 10.1007/9783-319-76900-4_9-1. (2019)

Khruengsai, Tanapichatsakul, S. y otros tres autores. Antifungal activity and chemical composition of endophytic fungus phanerochaete sp. MFLUCC16-0609', doi: 10.31925/farmacia.2019.4.8, Farmacia, 67(4), pp. 610-615. (2019).

Kitzberger, C., Smania, R., Pedroza, R., Ferreira S., Antioxidant and antimicrobial activities of shiitake (Lentinula edodes) extracts obtained by organic solvents and supercritical fluids', doi: 10.1016/j.jfoodeng.2006.06.013.Journal of Food Engineering, 80(2), pp. 631-638. (2007)

Kyekyeku, J. O., Kusari, S., y otros cinco autores. 'Antibacterial secondary metabolites from an endophytic fungus, Fusarium solani JK10', doi: 10.1016/j.fitote.2017.04.007 Fitoterapia, 119(February), pp. 108-114.. (2017) 
Liu, X.-B., Zheng, L y otros cuatro autores. Secondary metabolites form the endophytic fungus Fusarium equiseti and their antibacterial activities', doi: 10.1016/j.talanta.2010.09.042. Chemestry of Natural Compounds, 55(6), pp. 1141-1144. (2019)

Magalhães, L. M., Santos, F. y otros tres autores 'Rapid microplate high-throughput methodology for assessment of FolinCiocalteu reducing capacity', Talanta, 83(2), pp. 441-447. (2010)

Martínez, S. y Nakasone, K. K 'The genus Phanerochaete (Corticiaceae, Basidiomycotina) sensu lato in Uruguay', Sydowia, 57(1), pp. 94-101. (2005)

Montero-Recalde, M., Vayas L Evaluación de dos métodos para medir la sensibilidad de inhibición de crecimiento de la cepa certificada de Staphylococcus aureus subsp. aureus doi: 10.15381/rivep.v29i4.15185. Revista de Investigaciones Veterinarias del Perú, 29(4), p. 1543. (2018)

Orlandelli, R. C., Alberto R y otros tres autores. 'In vitro antibacterial activity of crude extracts produced by endophytic fungi isolated from piper hispidum sw.', doi: 10.7324/JAPS.2012.21027.Journal of Applied Pharmaceutical Science, 2(10), pp. 137-141. (2012)

Pansanit, A. y Pripdeevech, P. 'Antibacterial secondary metabolites from an endophytic fungus, Arthrinium sp. MFLUCC161053 isolated from Zingiber cassumunar' doi: 10.1080/21501203.2018.1481154., Mycology, 9(4), pp. 264-272. (2018)

Qi, J., Zhao, P. y otros cinco autores Anthraquinone Derivatives from a Sea Cucumber-Derived Trichoderma sp. Fungus with Antibacterial Activities', doi: 10.1007/s10600-020-02956-w. Chemistry of Natural Compounds, 56(1), pp. 112-114. (2020)

Ramirez-Aristizal L. S, Ortiz A, . Ospina, L. 'Evaluation of the antioxidant cpacity and characterization of phenolic compounds obatained from tea (Camellia sinenssis) for products pf diferents brands sold in Colombia', Pharmacology Online, 3 .pp 149.159 (2015)

Sahu, M. K, Kaushik., A, Das., H, Jha. 'In vitro and in silico antioxidant and antiproliferative activity of rhizospheric fungus Talaromyces purpureogenus isolate-ABRF2', doi: 10.1186/s40643-020-00303-z. (Bioresources and Bioprocessing, 7(1). 2020

Sharma, A., S, Kumari., V. Sharma., J, Saxena 'Production of extracellular lipase by submerged fermentation from fungal isolates of soil samples', Advance Pharmaceutical Journal 2(2), pp. 80-84. (2017)

Spirin, V., Volobuev, S y otros tres autores 'What is the type species of Phanerochaete (Polyporales, Basidiomycota)?', doi: 10.1007/s11557-016-1267-8. Mycological Progress, 16(2), pp. 171-183. (2017)

Suárez, C. 'Obtención in vitro de micelio de hongos comestibles, shiitake (lentinula edodes) y orellanas (Pleurotus ostreatus y Pleurotus pulmonarius) A PARTIR DE AISLAMIENTOS DE CUERPOS FRUCTÍFEROS, PARA LA PRODUCCIÓN DE SEMILLA', Universidad Nacional De Colombia, p. $86 . \quad$ Available at: http://www.bdigital.unal.edu.co/2792/.(2010)

Sulej, J., Osinaska, M., y otros seis autores. Antimicrobial and antioxidative potential of free and immobilised cellobiose dehydrogenase isolated from wood degrading fungi', doi: 10.1016/j.funbio.2019.09.007Fungal Biology, 123(12), pp. 875886.. (2019)

Vega, A., Franco, H. Productividad y calidad de los cuerpos fructíferos de los hongos comestibles Pleurotus pulmonarius RN2 y P. Djamor RN81 y RN82 cultivados sobre sustratos lignocelulósicos', doi: 10.4067/S0718-07642013000100009. Informacion Tecnologica, 24(1), pp. 69-78. (2013)

Yu, J. H., Keller, N. 'Regulation of secondary metabolism in filamentous fungi', doi: 10.1146/annurev.phyto.43.040204.140214. Annual Review of Phytopathology, 43(May 2014), pp. 437-458. (2005). 